\title{
The Westerbork Hydrogen Accretion in LOcal GAlaxieS (HALOGAS) survey
}

\section{Survey description and pilot observations}

\author{
G. Heald ${ }^{1}$, G. Józsa ${ }^{1}$, P. Serra ${ }^{1}$, L. Zschaechner ${ }^{2}$, R. Rand ${ }^{2}$, F. Fraternali ${ }^{3}$, T. Oosterloo ${ }^{1,4}$, R. Walterbos ${ }^{5}$, \\ E. Jütte ${ }^{6}$, and G. Gentile
}

\author{
${ }^{1}$ Netherlands Institute for Radio Astronomy (ASTRON), Postbus 2, 7990 AA Dwingeloo, The Netherlands \\ e-mail: heald@astron.nl \\ 2 University of New Mexico, 800 Yale Blvd, Albuquerque, NM, USA \\ 3 Astronomy Department, Bologna University, via Ranzani 1, 40127 Bologna, Italy \\ 4 Kapteyn Astronomical Institute, Postbus 800, 9700 AV Groningen, The Netherlands \\ 5 Department of Astronomy, New Mexico State University, PO Box 30001, MSC 4500, Las Cruces, NM 88003, USA \\ 6 Astronomisches Institut der Ruhr-Universität Bochum, Universitätsstr. 150, 44780 Bochum, Germany \\ Sterrenkundig Observatorium, Ghent University, Krijgslaan 281, S9, 9000 Ghent, Belgium
}

Received 15 October 2010 / Accepted 27 November 2010

\section{ABSTRACT}

\begin{abstract}
We introduce a new, very deep neutral hydrogen ( $\mathrm{H}$ ) survey being performed with the Westerbork Synthesis Radio Telescope (WSRT). The Westerbork Hydrogen Accretion in LOcal GAlaxieS (HALOGAS) Survey is producing an archive of some of the most sensitive $\mathrm{H}_{\text {I }}$ observations available, on the angular scales which are most useful for studying faint, diffuse gas in and around nearby galaxies. The survey data are being used to perform careful modeling of the galaxies, characterizing their gas content, morphology, and kinematics, with the primary goal of revealing the global characteristics of cold gas accretion onto spiral galaxies in the local Universe. In this paper, we describe the survey sample selection, the data acquisition, reduction, and analysis, and present the data products obtained during our pilot program, which consists of UGC 2082, NGC 672, NGC 925, and NGC 4565. The observations reveal a first glimpse of the picture that the full HALOGAS project aims to illuminate: the properties of accreting $\mathrm{H}_{\mathrm{I}}$ in different types of spirals, and across a range of galactic environments. None of the pilot survey galaxies hosts an $\mathrm{H}_{\mathrm{I}}$ halo of the scale of NGC 891 , but all show varying indications of halo gas features. We compare the properties of detected features in the pilot survey galaxies with their global characteristics, and discuss similarities and differences with NGC 891 and NGC 2403.
\end{abstract}

Key words. galaxies: spiral - galaxies: evolution - galaxies: ISM - galaxies: halos - galaxies: kinematics and dynamics

\section{Survey motivation}

It is becoming increasingly clear that gas accretion is a crucial piece of the puzzle of galaxy evolution. Accretion has been invoked as a possible solution to several problems in galactic astrophysics. A typical accretion rate of $\approx 1 M_{\odot} \mathrm{yr}^{-1}$ seems to be necessary in order for spiral galaxies to replenish gas consumed in the star formation process - it has been noted that otherwise, galaxies would use up their gas on timescales shorter than their ages (e.g., Larson et al. 1980). The constancy of the SFR in the solar neighborhood (Twarog 1980; Binney et al. 2000) argues that this inflow of fresh gas should locally be fairly constant. Similar accretion rates of metal-free gas may be needed to match the chemical evolution of the disk (e.g., Lacey \& Fall 1985; Schönrich \& Binney 2009). Accretion of fresh gas has also been invoked to explain the "G-dwarf problem", i.e. the small number of local stars with low metallicity (Pagel 1997), for instance by Chiappini et al. (1997). Large scale disturbances in galaxies, such as lopsidedness (both morphological and kinematical) and warps, may be the result of accretion (Bournaud et al. 2005; Sancisi et al. 2008). In their recent review of the observational evidence for cold accretion, Sancisi et al. (2008) infer a global average observed neutral gas accretion rate onto galaxies of $\gtrsim 0.2 M_{\odot} \mathrm{yr}^{-1}$. Such accretion may take place in two ways: through the merging of gas-rich satellites, and "pure" gas accretion from the intergalactic medium (IGM).

On theoretical grounds, a mode of accretion referred to as "cold" should continue in galaxies in low density environments through $z=0$ (e.g., Kereš et al. 2005). Note that this usage of the word "cold" differs from the meaning typically assumed by observers: in the theoretical picture of accretion, cold gas is that which is never shock heated to the virial temperature of the halo as it falls into the galactic potential, reaching maximum temperatures below $10^{5} \mathrm{~K}$ during the accretion process; observationally, cold gas means gas at temperatures up to nearly $10^{4} \mathrm{~K}$. Despite this difference in nomenclature, it seems clear that a significant portion of the accreting gas in local galaxies is expected to be at relatively low temperature, and that this gas phase will be traced, at least in part, by $\mathrm{H}_{\mathrm{I}}$.

Indeed, deep observations of a few nearby spirals - most notably the edge-on NGC 891 (Oosterloo et al. 2007), the moderately-inclined NGC 2403 (Fraternali et al. 2002), and the face-on NGC 6946 (Boomsma et al. 2008) - have shown that large quantities of cold gas exist in their halos, at large vertical distances from the thin disk. In the case of NGC 891, observations with the Westerbork Synthesis Radio Telescope (WSRT) revealed a huge neutral gas halo (e.g. Oosterloo et al. 2007, see 
their Fig. 1) which extends up to a vertical distance of $22 \mathrm{kpc}$, with massive filamentary structures far from the disk. In total, the Hi halo of NGC 891 contains almost $30 \%$ of the galaxy's total H I mass.

How can so much gas be located at such large vertical distances from the disk? Star formation-driven disk-halo flows, as described by galactic fountain (e.g., Bregman 1980) or chimney (Norman \& Ikeuchi 1989) models, are likely part of the explanation. The morphology of the extraplanar gas seems to be related to the properties of the underlying star forming disk (at least in its ionized phase; e.g., Rand 1996), and whether ionized gas is found in the halo or not seems to depend on the energy density provided by star formation (Rossa \& Dettmar 2003).

On the other hand, it has been shown that the rotational velocity of the halo gas (both in its neutral and ionized phases) decreases linearly with increasing distance from the midplane (Fraternali et al. 2005; Heald et al. 2006; Kamphuis et al. 2007). This kinematic "lag" - which allows us to distinguish halo gas from disk gas in moderately inclined galaxies such as NGC 2403 - is much steeper than can be accounted for with simple galactic fountain models (the discrepancy is observed to be a factor of two or more; Heald et al. 2007). A good deal of theoretical effort has been put into this problem, and a range of more sophisticated models to explain the kinematics of gaseous halos have been put forward (using $\mathrm{N}$-body+smoothed particle hydrodynamic (SPH) simulations, Kaufmann et al. (2006); hydrostatic models, Benjamin (2000); Barnabè et al. (2006); Marinacci et al. (2010b); SPH including spiral arms and bars, Struck \& Smith (2009); and cloud-corona interaction, Marinacci et al. (2010a)). Such efforts have shown how the steep lag in NGC 891 might occur. Additionally, a constant accretion of low-angular momentum gas has been invoked, in combination with the simple galactic fountain picture, to slow the halo gas (the required accretion rate would be $\approx 3 M_{\odot} \mathrm{yr}^{-1}$ in NGC 891 based on modeling work by Fraternali \& Binney (2008) - in this picture, $90 \%$ of the halo gas originates from galactic fountain, and 10\% from accretion). Distinguishing between these possible models requires a larger sample of gaseous halos and measurements of their kinematics.

It has been pointed out that if the largest $\mathrm{H}_{\mathrm{I}}$ filament in the halo of NGC 891 was created by disk star formation, an energy equivalent to $\sim 10^{5}$ supernovae would be required. This suggests an external origin instead (Oosterloo et al. 2007). A final piece of evidence suggestive of accretion in NGC 891 comes in the form of small, $10^{6} M_{\odot}$ clouds which are counter-rotating in the halo. A filament with a mass of $10^{7} M_{\odot}$ is found in NGC 2403 (Fraternali et al. 2002), and a counter-rotating $\sim 10^{7} M_{\odot}$ cloud is also detected in the extraplanar region of NGC 5746 (Rand \& Benjamin 2008).

Tracers of ongoing accretion can also be found closer to home: many of the MW's high velocity clouds (HVCs) have low metallicity (Tripp et al. 2003); together with the increasingly tight constraints on their distances (Wakker et al. 2007, 2008), the evidence is consistent with the idea that at least part of the HVC population is infalling primordial gas. HVC analogs have also been detected in the outskirts of M31 (Thilker et al. 2004). In both the MW and M 31, stellar streams are observed via deep optical observations (Belokurov et al. 2007; Ferguson 2007). The H I filament in the halo of NGC 891 may be the cold gas counterpart of this type of stream. It has been recently recognized that NGC 891 also has a giant stellar stream looping around the disk, pointing to recent interaction (Mouhcine et al. 2010). Observing such features in $\mathrm{H}_{\mathrm{I}}$ emission has the advantage that we directly obtain kinematical information.
Halo gas is also present in at least one low surface brightness (LSB) galaxy (NGC 4395) with very little ongoing star formation, and a $10^{7} M_{\odot}$ gas cloud in that system is likely in the process of being accreted (Heald \& Oosterloo 2008). Moreover, evidence suggests the presence of a neutral gas halo in the superthin LSB UGC 7321 (Matthews \& Wood 2003). On the other hand, there is no evidence for large amounts of $\mathrm{H}$ I in the halo of the very massive NGC 5746 (Rand \& Benjamin 2008).

Why do some galaxies have massive gaseous halos, but not others? Is the Hi halo of NGC 891 typical or exceptional? At present, the extremely limited number of sufficiently deep observations restricts our ability to draw general conclusions about the prevalence of accretion in the local Universe. A comprehensive investigation is needed, using a representative sample of spiral galaxies. Most of the existing deep observations were prompted by earlier shallow observations which already showed evidence for an exceptional halo population or other peculiarities. Moreover, the targeted galaxies were chosen individually, rather than as a part of a larger program to sample a wide range of galactic disk properties such as star formation rates, warps, and lopsidedness.

As a first attempt to provide such information, we have started the Westerbork Hydrogen Accretion in LOcal GAlaxieS (HALOGAS) Survey. With this project we have the goal to make a significant step forward in understanding the process of cold gas accretion in spiral galaxies, by investigating the general phenomenon for the first time.

The HALOGAS Survey observations are designed to detect and characterize neutral extraplanar gas by measuring its total mass, rotation speed (in edge-ons), radial inflow or outflow (in inclined galaxies such as NGC 2403; see the work by Fraternali et al. 2002), and distribution (smooth, filamentary, or clumpy?). Deriving the extraplanar gas kinematics is of crucial importance - these parameters are key predictions of halo gas and accretion models. Comparisons between the extraplanar gas characteristics and the properties of the spiral disk should clarify the roles of star formation and accretion.

The HALOGAS data will form a publicly available repository of the deepest $\mathrm{H}_{\mathrm{I}}$ observations of nearby spiral galaxies. Additional studies which will be enabled by this dataset are numerous, and include:

1. search for background continuum source variability at low ( 1 mJy) flux levels, over the full HALOGAS survey area (totalling $\sim 6.2$ square degrees within the WSRT primary beam $F W H M$ );

2. detailed analyses of the gravitational potential by measuring rotation curves extending far into the faint outer disks, and additionally by using the combination of the gas dispersion and vertical scaleheight;

3. studies of warps and lopsidedness in spiral galaxies; and

4. the details of the H I cutoff at the edge of the gaseous disk, at sensitivity levels comparable to the requirement specified by e.g. Corbelli \& Bandiera (2002) to examine the $\mathrm{H}_{\text {I }}-\mathrm{H}_{\text {II }}$ transition.

The survey observations are being completed at the WSRT. Our first semester of observations was designed as a small pilot program, and that pilot survey is the focus of this paper.

The paper is organized as follows. We describe the selection of the survey sample in Sect. 2. The observational setup and data reduction path are outlined in Sect. 3. Results from the HALOGAS pilot survey are presented in Sect. 4. We discuss the first results from the HALOGAS Survey in Sect. 5. Finally, 
multi-wavelength supplements to the HALOGAS Survey, and our data release plans, are discussed in Sects. 6 and 7.

\section{The HALOGAS sample}

To reach the sensitivity required to detect and characterize faint, diffuse $\mathrm{H}_{\mathrm{I}}$, with characteristic column densities of a few times $10^{19} \mathrm{~cm}^{-2}$, each of the HALOGAS targets is being observed at the WSRT for a total of $10 \times 12 \mathrm{~h}$. We would like to have an unbiased look at the accretion process in the local Universe. From this perspective, non-detections of extraplanar gas are as interesting as the detections - by restricting attention to galaxies already suspected to show clear signs of accretion, we would learn less about the ubiquity of the accretion process in the spiral population, or the relationship between galaxy parameters and the accretion process. We therefore deliberately avoided selecting or rejecting sample galaxies based on existing $\mathrm{H}_{\mathrm{I}}$ observations, or other subjective judgements about how interesting a target might appear when considered as an individual target. The final sample of galaxies was, ultimately, chosen on the basis of a small number of basic and neutral selection criteria, each of which are designed to maximize our ability to detect and study the properties of any diffuse gas which might be present in the target systems.

To select the galaxies for our sample, we searched in the Tully (1988) catalog, requiring the following characteristics:

(i) the sample is made up of barred and unbarred spirals with Hubble types between $\mathrm{Sa}$ and $\mathrm{Sd}$;

(ii) because the WSRT is an east-west interferometer, we require that the source declination $\delta>+25^{\circ}$ to ensure that the synthesized beamsize is $\lesssim 30^{\prime \prime}$;

(iii) we only select nearby galaxies with distance $D<11 \mathrm{Mpc}$, in order to observe at adequate physical resolution to resolve the morphology of the galaxies (for instance, the characteristic WSRT beamsize of $15^{\prime \prime}=0.7 \mathrm{kpc}$ at a target distance of $10 \mathrm{Mpc}$ );

(iv) in order to ensure that each galaxy is sampled by a reasonably large number of angular resolution elements, we require a large optical isophotal diameter at 25th magnitude $D_{25}>3^{\prime}$. Typically, $\mathrm{H}_{\mathrm{I}}$ disks are larger than $D_{25}$;

(v) finally, we require that the systemic velocity is $>100 \mathrm{~km} \mathrm{~s}^{-1}$, in order to avoid confusion with MW H I.

Within these criteria, we selected all intermediately inclined $\left(50^{\circ} \leq i \leq 75^{\circ}\right)$ and edge-on $\left(i \geq 85^{\circ}\right)$ galaxies. Galaxies in the former category reveal connections between halo gas and star formation, and allow investigation of rotation and radial motions in the halo gas (e.g. Fraternali et al. 2002); edge-on galaxies are useful for learning about the extent of halos, and the vertical change in rotation speed of the halo gas (e.g. Oosterloo et al. 2007). Visual inspection was utilized only to discard galaxies with incorrectly catalogued inclination angles. We also excluded from our new observations two galaxies which meet our criteria, but which are already deeply observed in H I (namely, NGC 891 and NGC 2403). We will incorporate the properties of these galaxies into our final statistical sample since they match the sample criteria described above. The final sample of 24 galaxies is listed in Table 1. The columns are (1) the UGC ID; (2) NGC or Messier IDs if available; (3) the Hubble type; (4) the distance $d_{\mathrm{T} 88}$ in Mpc from Tully (1988); (5) the adopted distance $d_{\text {best }}$ in Mpc; (6) the method for determining $d_{\text {best }}$; and our best estimate using the tabulated method; (7) the systemic velocity $v_{\text {sys }}$ in $\mathrm{km} \mathrm{s}^{-1}$; (8) the inclination $i$; (9) the optical diameter $D_{25} ;(10)$ the blue magnitude $M_{B} ;(11)$ the rotational velocity $v_{\text {rot }} ;(12)$ the star formation rate (SFR); and (13) references used in deriving the SFR values in Col. (12), see below for details. The UGC IDs of the four targets which have been observed in the pilot survey described in this paper are indicated with a bold font.

The galaxy parameters listed in Table 1 are taken from Tully (1988), except for $v_{\text {rot }}$, which is from HyperLEDA (Paturel et al. 2003), the adopted distance $d_{\text {best }}$, and the SFR, both of which are discussed below. Given the broad range of Hubble type, SFR, mass, and environmental parameters (see Table 2 and the discussion below) exhibited by nearby spiral galaxies, this sample size stikes an acceptable balance between the number of targets required to reasonably sample the parameter space, while still resulting in a total amount of observing time which does not become prohibitive.

One of the sample selection criteria is to select only galaxies closer than $11 \mathrm{Mpc}$. This selection is based on galaxy distances taken from Tully (1988), which we indicate as $d_{\text {T88. }}$. More recent distance estimates are available for all selected galaxies. Adopting the best one is important when measuring, e.g., mass and linear size of the detected $\mathrm{H}_{\mathrm{I}}$ structures. Therefore, we searched the NASA/IPAC Extragalactic Database (NED) ${ }^{1}$ distance database to obtain the most accurate distance of each galaxy, which we indicate as $d_{\text {best }}$. Table 1 lists both $d_{\mathrm{T} 88}$ and $d_{\text {best }}$ for all galaxies in the sample. We define $d_{\text {best }}$ using one of the following methods, sorted according to decreasing priority, depending on which measurements are available:

1. median of all Cepheid and/or tip of the red-giant branch distances: used for 10 galaxies;

2. median of all distance estimates obtained with any of the following methods: planetary-nebulae luminosity function, brightest stars, radio and optical SNII, masers, globularcluster luminosity function: used for 5 galaxies;

3. median of all Tully-Fisher distances (including $d_{\mathrm{T} 88}$ ): used for 8 galaxies;

4. Tully (1988) estimate: used for 1 galaxy;

It is worth noting that $d_{\text {best }}$ is significantly different from $d_{\mathrm{T} 88}$ ( $>2 \mathrm{Mpc}$ ) in about a third of all galaxies. For NGC 3198 and NGC $4414 d_{\text {best }}$ is obtained with method 1, and is respectively 3.7 and 8.1 Mpc larger than $d_{\mathrm{T} 88}$. For NGC 4062, NGC 4274, UGC 2082, UGC 4278 and UGC 7774, $d_{\text {best }}$ is obtained with method 3 and is always larger than $d_{\mathrm{T} 88}$ (the last galaxy is the most extreme case, with a difference of $17.6 \mathrm{Mpc}$ between the two estimates). In general, $d_{\mathrm{T} 88}$ is always one of the lowest (when not the lowest) distance estimates available.

The method that we use to calculate star formation rates also bears some discussion. The galaxies that make up the HALOGAS sample are not otherwise included in a uniform observational sample at other wavelengths, in particular the infrared. We therefore use different methods for individual targets to calculate SFR, depending on what data are available. We prefer to use the combination of $\mathrm{H} \alpha$ and TIR luminosity given by Kennicutt et al. (2009), where $L_{\mathrm{TIR}}$ is either calculated from IRAS or Spitzer fluxes via the prescriptions given by Dale \& Helou (2002). The IRAS form is used where possible, and the Spitzer form where IRAS fluxes are not available or provide only upper limits for the $25 \mu \mathrm{m}$ flux. IRAS fluxes are obtained from the references listed in Table 1, and the Spitzer fluxes are from the Local Volume Legacy (LVL; Dale et al. 2009). H $\alpha$ fluxes are

1 The NASA/IPAC Extragalactic Database (NED) is operated by the Jet Propulsion Laboratory, California Institute of Technology, under contract with the National Aeronautics and Space Administration. 
Table 1. The full HALOGAS sample.

\begin{tabular}{|c|c|c|c|c|c|c|c|c|c|c|c|c|}
\hline UGC & Other IDs & Type & $\begin{array}{c}d_{\mathrm{T} 88} \\
(\mathrm{Mpc})\end{array}$ & $\begin{array}{c}d_{\text {best }} \\
(\mathrm{Mpc})\end{array}$ & $\begin{array}{c}d_{\text {best }} \\
\text { method }\end{array}$ & $\begin{array}{c}v_{\text {sys }} \\
\left(\mathrm{km} \mathrm{s}^{-1}\right)\end{array}$ & $\begin{array}{c}i \\
\left(^{\circ}\right)\end{array}$ & $\begin{array}{c}D_{25} \\
\text { (arcmin) }\end{array}$ & $\begin{array}{c}M_{B} \\
(\mathrm{mag})\end{array}$ & $\begin{array}{c}v_{\text {rot }} \\
\left(\mathrm{km} \mathrm{s}^{-1}\right)\end{array}$ & $\begin{array}{c}S F R \\
\left(M_{\odot} \mathrm{yr}^{-1}\right)\end{array}$ & $\begin{array}{l}S F R \\
\text { Refs. }\end{array}$ \\
\hline 1256 & NGC 0672 & SBcd & 7.5 & 7.6 & 2 & 425 & 70 & 6.4 & -18.65 & 130.7 & 0.22 & $\mathrm{H} 1, \mathrm{I} 2$ \\
\hline 1831 & NGC 0891 & $\mathrm{SAb}$ & 9.6 & 9.2 & 1 & 529 & 84 & 12.2 & -19.96 & 212.2 & 2.4 & $\mathrm{H} 1, \mathrm{I} 4$ \\
\hline 1913 & NGC 0925 & SABd & 9.4 & 9.1 & 1 & 554 & 54 & 11.3 & -19.66 & 102.4 & 0.83 & $\mathrm{H} 1, \mathrm{I} 4$ \\
\hline 1983 & NGC 0949 & SAd & 10.3 & 11.3 & 2 & 610 & 52 & 3.5 & -17.85 & 90.9 & 0.26 & H1, I1 \\
\hline 2082 & - & SAc & 10.7 & 14.4 & 3 & 710 & 89 & 5.8 & -18.55 & 86.6 & 0.023 &,$- \mathrm{I} 1$ \\
\hline 2137 & NGC 1003 & SAcd & 10.7 & 11.6 & 3 & 626 & 67 & 6.3 & -18.61 & 95.5 & 0.34 & $\mathrm{H} 1, \mathrm{I} 2$ \\
\hline 3918 & NGC 2403 & SAcd & 4.2 & 3.2 & 1 & 132 & 62 & 23.8 & -19.68 & 121.9 & 1.0 & $\mathrm{H} 1, \mathrm{I} 4$ \\
\hline 4278 & IC 2233 & SAd & 10.6 & 13.6 & 3 & 565 & 90 & 4.3 & -17.45 & 79.2 & 0.11 & H1, I5 \\
\hline 4284 & NGC 2541 & SAcd & 10.6 & 12.0 & 1 & 553 & 67 & 7.2 & -18.37 & 92.1 & $<0.27^{a}$ & $\mathrm{H} 2, \mathrm{I} 2$ \\
\hline 5572 & NGC 3198 & $\mathrm{SBc}$ & 10.8 & 14.5 & 1 & 660 & 71 & 8.8 & -19.62 & 148.2 & 0.61 & $\mathrm{H} 2, \mathrm{I} 4$ \\
\hline 7045 & NGC 4062 & SAc & 9.7 & 16.9 & 3 & 769 & 68 & 4.5 & -18.27 & 140.5 & 0.22 & $\mathrm{H} 2, \mathrm{I} 2$ \\
\hline 7322 & NGC 4244 & SAcd & 3.1 & 4.4 & 1 & 247 & 90 & 15.8 & -17.60 & 89.0 & 0.058 & H1, I5 \\
\hline 7353 & NGC 4258 (M 106) & SABbc & 6.8 & 7.6 & 1 & 449 & 71 & 17.1 & -20.59 & 208.0 & 1.4 & $\mathrm{H} 1, \mathrm{I} 3$ \\
\hline 7377 & NGC 4274 & SBab & 9.7 & 19.4 & 3 & 922 & 72 & 6.5 & -19.22 & 239.9 & 0.31 & $\mathrm{H} 3, \mathrm{I} 2$ \\
\hline 7539 & NGC 4414 & SAc & 9.7 & 17.8 & 1 & 720 & 50 & 4.5 & -19.12 & 224.7 & 1.3 & $\mathrm{H} 2, \mathrm{I} 4$ \\
\hline $7591^{b}$ & NGC 4448 & SBab & 9.7 & 9.7 & 4 & 693 & 71 & 3.8 & -18.43 & 221.6 & 0.056 &,$- \mathrm{I} 2$ \\
\hline 7766 & NGC 4559 & SABcd & 9.7 & 7.9 & 3 & 816 & 69 & 11.3 & -20.07 & 113.4 & 1.1 & $\mathrm{H} 4, \mathrm{I} 4$ \\
\hline 7772 & NGC 4565 & SAb & 9.7 & 10.8 & 2 & 1228 & 90 & 16.2 & -20.34 & 244.9 & 0.54 & $\mathrm{H} 5, \mathrm{I} 4$ \\
\hline $7774^{b}$ & - & SAd & 6.8 & 24.4 & 3 & 526 & 90 & 3.5 & -15.57 & 79.4 & 0.0074 & $\mathrm{H} 1, \mathrm{I} 5$ \\
\hline 7865 & NGC 4631 & SBd & 6.9 & 7.6 & 1 & 613 & 85 & 14.7 & -20.12 & 138.9 & 2.1 & $\mathrm{H} 1, \mathrm{I} 4$ \\
\hline 8286 & NGC 5023 & SAc & 6.0 & 6.6 & 1 & 400 & 90 & 6.8 & -17.29 & 80.3 & 0.032 & H1, I5 \\
\hline 8334 & NGC 5055 (M 63) & SAbc & 7.2 & 8.5 & 3 & 497 & 55 & 13.0 & -20.14 & 215.5 & 1.5 & $\mathrm{H} 1, \mathrm{I} 4$ \\
\hline 8550 & NGC 5229 & $\mathrm{SBc}$ & 6.4 & 5.1 & 2 & 365 & 90 & 3.5 & -15.82 & 57.3 & 0.014 & $\mathrm{H} 1, \mathrm{I} 5$ \\
\hline 9179 & NGC 5585 & SABd & 7.0 & 8.7 & 2 & 303 & 51 & 5.5 & -17.96 & 79.1 & 0.26 & $\mathrm{H} 1, \mathrm{I} 1$ \\
\hline
\end{tabular}

Notes. ${ }^{(a)}$ The SFR value for NGC 2541 is strictly speaking an upper limit, because the IRAS $25 \mu \mathrm{m}$ flux is catalogued as a non-detection by Moshir et al. (1990). ${ }^{(b)}$ No H I observing time granted yet.

References. (H1) Kennicutt et al. (2008); (H2) Moustakas \& Kennicutt (2006); (H3) Hameed \& Devereux (2005); (H4) Kennicutt et al. (2009); (H5) Robitaille et al. (2007); (I1) Lisenfeld et al. (2007); (I2) Moshir et al. (1990); (I3) Rice et al. (1988); (I4) Sanders et al. (2003); (I5) Dale et al. (2009).

obtained first from Kennicutt et al. (2008), and for targets not included in that work, the literature was searched for fluxes. In all cases luminosities are calculated using the adopted distances listed in Table 1. In two cases, UGC 2082 and NGC 4448, no H $\alpha$ fluxes are available; but since IRAS $60 \mu \mathrm{m}$ and $100 \mu \mathrm{m}$ fluxes are available, we calculate $L_{\mathrm{FIR}}$ using the equation given by Rice et al. (1988), and use SFR $\left(L_{\mathrm{FIR}}\right)$ as described by Kennicutt (1998). The references given in Table 1 were used to obtain the $\mathrm{H} \alpha$ and IR fluxes. The derivations of the SFR for the HALOGAS sample will become more uniform with the release of the Widefield Infrared Survey Explorer (WISE; Wright 2008) data, which will also be well matched in angular resolution to our WSRT observations.

In Table 2, we show environmental information about the target galaxies. The data are from the group catalog developed from the Nearby Optical Galaxies (NOG) sample (Giuricin et al. 2000). The columns in the table are: (1) galaxy ID; (2) number of group members (using the percolation-1 grouping algorithm, see below); (3) NOG group (NOGG) number; (4) total group $B$ luminosity; (5) distance from group center of mass to outermost member; $(6,7)$ minimum and maximum group member velocity relative to group center of mass; (8) contribution of the target to the group $B$ luminosity; (9) fraction of maximum group member $B$ luminosity; (10) normalized distance to group center of mass; (11) target velocity relative to group center of mass.

We have chosen to use group definitions resulting from the Giuricin et al. (2000) "P1" algorithm (which is a percolation friends-of-friends algorithm that keeps the velocity grouping condition fixed irrespective of the galaxies' redshift). Because this definition of groups is based on angular and velocity coincidences, there will be spurious groupings or separations. We note corrections to the group definitions where appropriate in the text, and otherwise use the information as a means to illustrate the overall environmental characteristics of the HALOGAS sample. The survey galaxies sample a fairly broad range in environment. One quarter (6/24) of the HALOGAS sample reside in the same large group (Coma I). Three targets are members of pairs. In only one case is there a galaxy with a strongly interacting neighbor: NGC 672, which was included in the pilot survey described in this paper. The range of galaxy properties with respect to the group to which they belong also exhibits a wide range of values. The HALOGAS sample contains galaxies which dominate their group, as well as those which are minor group members and companions. If these smaller targets are gas rich, we may be probing gas accretion donors as well as recipients. Considering all of these properties, after observations of the full HALOGAS sample, we expect to be able to assess whether there is an environmental dependence on target galaxy halo properties.

Because the HALOGAS galaxies are all nearby, there is the added advantage that most have been extensively observed at other wavelengths, for example as part of recent surveys such as the Spitzer Infrared Nearby Galaxies Survey (SINGS; Kennicutt et al. 2003) and the $11 \mathrm{Mpc} \mathrm{H} \alpha$ and Ultraviolet Galaxy Survey (11HUGS; Lee et al. 2007). The star formation process is clearly of interest with relation to the extraplanar, halo, and accreting gas that we are tracing, and we will therefore benefit by having these data available for our investigation. Although the available data will help to characterize disk star formation rates, we note 
G. Heald et al.: The WSRT HALOGAS survey. I.

Table 2. Environmental properties of the HALOGAS sample galaxies.

\begin{tabular}{|c|c|c|c|c|c|c|c|c|c|c|}
\hline $\begin{array}{l}\text { Galaxy } \\
\text { ID }\end{array}$ & $\begin{array}{l}\text { \# Group } \\
\text { members }\end{array}$ & $\begin{array}{l}\text { NOGG } \\
\text { number }\end{array}$ & $\begin{array}{c}L_{B, \text { group }} \\
{\left[10^{10} M_{\odot}\right]}\end{array}$ & $\begin{array}{l}R_{\text {group }} \\
{[\mathrm{Mpc}]}\end{array}$ & $\begin{array}{l}v_{\min , \text { group }} \\
{\left[\mathrm{km} \mathrm{s}^{-1}\right]}\end{array}$ & $\begin{array}{l}v_{\max } \text { group } \\
{\left[\mathrm{km} \mathrm{s}^{-1}\right]}\end{array}$ & $L_{B} / L_{B, \text { group }}$ & $L_{B} / L_{B, \max }$ & $R / R_{\text {group }}$ & {$\left[\begin{array}{c}v \\
{\left[\mathrm{~km} \mathrm{~s}^{-1}\right]}\end{array}\right.$} \\
\hline NGC 0672 & 2 & 88 & 1.3 & 0.01 & -52 & 29 & 0.64 & 1.00 & 0.55 & 29 \\
\hline NGC $0891^{a}$ & - & - & - & - & - & - & - & - & - & - \\
\hline NGC 0925 & 6 & 149 & 3.1 & 0.56 & -16 & 47 & 0.69 & 1.00 & 0.25 & -16 \\
\hline NGC 0949 & 6 & 149 & 3.1 & 0.56 & -16 & 47 & 0.15 & 0.21 & 1.00 & 47 \\
\hline UGC $2082^{b}$ & - & - & - & - & - & - & - & - & 1.00 & - \\
\hline NGC $1003^{a}$ & - & - & - & - & - & - & - & - & - & - \\
\hline NGC 2403 & 2 & 319 & 1.8 & 0.24 & -14 & 1 & 0.88 & 1.00 & 0.13 & 1 \\
\hline UGC 4278 & 6 & 345 & 1.4 & 0.77 & -90 & 35 & 0.30 & 1.00 & 0.44 & 21 \\
\hline NGC 2541 & 6 & 345 & 1.4 & 0.77 & -90 & 35 & 0.24 & 0.79 & 0.21 & 35 \\
\hline NGC 3198 & 2 & 462 & 1.7 & 0.43 & -25 & 1 & 0.96 & 1.00 & 0.05 & 1 \\
\hline NGC 4062 & 27 & $631^{c}$ & 21.0 & 1.27 & -163 & 525 & 0.03 & 0.07 & 0.86 & 45 \\
\hline NGC 4244 & 16 & 636 & 3.1 & 0.80 & -93 & 83 & 0.22 & 0.66 & 0.31 & -42 \\
\hline NGC 4258 & 21 & 644 & 9.0 & 1.07 & -103 & 160 & 0.51 & 1.00 & 0.24 & -40 \\
\hline NGC 4274 & 27 & $631^{c}$ & 21.0 & 1.27 & -163 & 525 & 0.04 & 0.10 & 0.45 & 194 \\
\hline NGC 4414 & 27 & $631^{c}$ & 21.0 & 1.27 & -163 & 525 & 0.05 & 0.13 & 0.21 & -3 \\
\hline NGC 4448 & 27 & $631^{c}$ & 21.0 & 1.27 & -163 & 525 & 0.02 & 0.06 & 0.37 & -79 \\
\hline NGC 4559 & 27 & $631^{c}$ & 21.0 & 1.27 & -163 & 525 & 0.12 & 0.30 & 0.42 & 79 \\
\hline NGC 4565 & 3 & 648 & 14.9 & 0.26 & -18 & 107 & 0.78 & 1.00 & 0.25 & -18 \\
\hline UGC 7774 & 21 & 644 & 9.0 & 1.07 & -103 & 160 & 0.01 & 0.01 & 0.74 & 3 \\
\hline NGC 4631 & 27 & $631^{c}$ & 21.0 & 1.27 & -163 & 525 & 0.40 & 1.00 & 0.30 & -91 \\
\hline NGC 5023 & 7 & 723 & 8.0 & 0.45 & -94 & 131 & 0.04 & 0.09 & 0.68 & -77 \\
\hline NGC 5055 & 7 & 723 & 8.0 & 0.45 & -94 & 131 & 0.37 & 0.79 & 1.00 & 8 \\
\hline NGC 5229 & 7 & 723 & 8.0 & 0.45 & -94 & 131 & 0.01 & 0.02 & 0.97 & -94 \\
\hline NGC 5585 & 8 & 766 & 3.1 & 0.54 & 107 & 75 & 0.07 & 0.09 & 0.52 & 75 \\
\hline
\end{tabular}

Notes. ${ }^{(a)}$ Not included in the Giuricin et al. (2000) catalog due to low galactic latitude. ${ }^{(b)}$ No environmental information available; UGC 2082 is isolated. ${ }^{(c)}$ NOGG number 631 corresponds to Coma I.

that the coverage is not enough to cover the full WSRT primary beam. Furthermore, we are pursuing deep observations at other observational facilities to fill gaps where necessary (see Sect. 6).

\section{Observations and data reduction}

The data are being obtained using the WSRT, which is an eastwest interferometer consisting of 10 fixed (of which one is not used) and 4 movable 25-m antennas. Baselines range from $36 \mathrm{~m}$ to $2.7 \mathrm{~km}$, and the fixed antennas are on a regular grid with a spacing of $144 \mathrm{~m}$. We used the array in its Maxishort configuration, which is designed to optimize the imaging performance for extended sources in individual tracks. The correlator was set up to provide two linear polarizations in 1024 channels over a $10 \mathrm{MHz}$ bandwidth centered at the systemic velocity of each target. Each galaxy was observed for 10 full 12-h tracks, and each track was bracketed by standard flux calibrator sources.

Data reduction was performed using usual techniques in the Miriad software package (Sault et al. 1995). A standard data reduction path was used for most targets; exceptions were necessary due to individual circumstances and will be described where appropriate. First, the raw data were visually inspected, and samples corrupted by radio frequency interference (RFI) were flagged where necessary. System temperature variations, which are measured and recorded at each antenna during the observations, were corrected for in both the calibrator and target data sets. Next, the standard calibrator sources were used to set the flux scale, as well as define and correct for the bandpass response. The radio continuum emission in the field was subtracted in the visibility domain and saved as "channel-0" data. An imaging and self-calibration loop was used on the continuum data to solve for time-variable antenna gain phases. The resulting antenna gains were subsequently applied to the continuum-subtracted spectral line data. The H I data were imaged with a variety of weighting schemes (described below), creating several different data cubes for each target (see Table 3). Offline Hanning smoothing led to a final velocity resolution of $4.12 \mathrm{~km} \mathrm{~s}^{-1}$, although we may choose to degrade this resolution in post-processing in order to maximize sensitivity to features with broad velocity width. Finally, Clark CLEAN deconvolution was performed in two stages, the first within masked regions containing the brightest line emission, and the second within the entire imaged region.

Different weighting schemes are used in inverting the data from the $u, v$ plane into the image domain. In practice, within miriad's invert task, we use a robust parameter of 0 for intermediate resolution and sensitivity. To maximize sensitivity to faint extended emission we additionally use a Gaussian $u, v$ taper corresponding to $30^{\prime \prime}$ in the image plane. See Figs. 1, 3, 5, and 7.

For the pilot survey galaxies presented in this paper, we list in Table 3 a summary of the data cubes which have been produced and analyzed for the purposes of the presentation in Sect. 4. The columns are (1) Galaxy ID; (2) cube description; $(3,4)$ beam size in arcsec and in kpc (note, the beam position angle is in all cases very close to $0^{\circ}$ because WSRT is an east-west array); (5) rms noise level; (6) the $3 \sigma$ column density sensitivity to emission with a velocity width of $16 \mathrm{~km} \mathrm{~s}^{-1}$; and (7) the

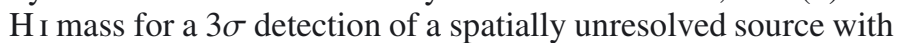
the same $16 \mathrm{~km} \mathrm{~s}^{-1}$ velocity width. The $\mathrm{H}_{\mathrm{I}}$ masses are calculated using $d_{\text {best }}$ from Table 1 .

The data cubes were used to generate images of the total $\mathrm{H}_{\mathrm{I}}$ line intensity (moment- 0 images), and of the intensity-averaged line-of-sight velocity (first-moment images). These were produced by smoothing the data cubes to a resolution of $90^{\prime \prime}$, clipping the original cubes at the $3 \sigma$ level of the smoothed cubes, and performing the moment calculations mentioned above. The 
Table 3. Properties of pilot survey datacubes.

\begin{tabular}{llccccc}
\hline \hline $\begin{array}{l}\text { Galaxy } \\
\text { ID }\end{array}$ & $\begin{array}{l}\text { Visibility } \\
\text { weighting }\end{array}$ & $\begin{array}{c}\text { Beam size } \\
(\operatorname{arcsec})\end{array}$ & $\begin{array}{c}\text { Beam size } \\
(\mathrm{kpc})\end{array}$ & $\begin{array}{c}\text { rms noise } \\
\left(\mathrm{mJy} \mathrm{beam}^{-1}\right)\end{array}$ & $\begin{array}{c}3 \sigma \text { column density } \\
\text { sensitivity }\left(10^{19} \mathrm{~cm}^{-2}\right)\end{array}$ & $\begin{array}{c}3 \sigma \mathrm{H}_{\text {I mass }} \\
\text { sensitivity }\left(10^{5} M_{\odot}\right)\end{array}$ \\
\hline UGC 2082 & uniform & $24.4 \times 11.3$ & $1.7 \times 0.8$ & 0.28 & 5.4 & 6.6 \\
& robust-0 & $29.1 \times 12.6$ & $2.0 \times 0.9$ & 0.21 & 3.0 & 4.9 \\
NGC 672 & 30" taper & $40.9 \times 33.1$ & $2.9 \times 2.3$ & 0.20 & 0.8 & 4.7 \\
& uniform & $27.4 \times 12.3$ & $1.0 \times 0.5$ & 0.28 & 4.4 & 1.8 \\
& robust-0 & $30.6 \times 13.5$ & $1.1 \times 0.5$ & 0.21 & 2.7 & 1.4 \\
NGC 925 & 30" taper & $41.7 \times 32.0$ & $1.5 \times 1.2$ & 0.19 & 0.8 & 2.3 \\
& uniform & $22.2 \times 12.6$ & $1.0 \times 0.6$ & 0.24 & 4.5 & 1.7 \\
& robust-0 & $25.3 \times 13.9$ & $1.1 \times 0.6$ & 0.18 & 0.7 & 3.6 \\
NGC 4565 & 30" taper & $37.9 \times 33.2$ & $1.7 \times 1.5$ & 0.17 & 3.9 & 2.3 \\
& uniform & $27.4 \times 12.0$ & $1.4 \times 0.6$ & 0.24 & 2.0 & 3.0 \\
& robust-0 & $31.9 \times 13.8$ & $1.7 \times 0.7$ & 0.17 & 0.8 & \\
\hline
\end{tabular}

miriad task moment was used for this process. In the case of the total intensity map, the primary beam correction was applied (for the WSRT 25-m antennas, the primary beam shape takes the mathematical form $\cos ^{6}(c r v)$, where $c$ is a constant with a value of 68 at $L$-band, $v$ is the frequency in $\mathrm{GHz}$, and $r$ is the field radius in radians). Then, intensities were converted to column densities $\left(N_{\mathrm{HI}}\right)$ assuming optically thin gas. The resulting maps are displayed and discussed in Sect. 4. We note that typical velocity profiles are more complicated than can be encapsulated in a single number such as the average velocity. Especially in edgeon galaxies, first-moment maps can be misleading, and therefore for our two highly inclined targets we instead show Renzograms (a single outer contour for each of a series of velocity channels, plotted on the same map).

The primary goal of the survey is to characterize each galaxy in the sample by the properties of the extraplanar gas (if any) detected in the field, and to consider the full sample together to draw conclusions about the global characteristics of this phenomenon. A detailed picture of the individual galaxies will be one of the output products of this investigation. The results for such individual survey targets will be presented in a series of companion papers. The main engine for analyzing these galaxies will be tilted-ring modeling software, which builds geometrical galaxy models using a series of idealized, roughly concentric, tilted rings. The tilted-ring modeling software is based in the GIPSY package. We will use a combination of GIPSY's GALMOD, and the Tilted Ring Fitting Code (TiRiFiC) which has been developed by Józsa et al. (2007).

\section{Overview of pilot survey observations}

In this section, we present an overview of the data obtained during our pilot survey, which is made up of four targets observed during the first observing semester of the HALOGAS survey. The targets presented here are representative of a broad range of galaxy characteristics, and give a sense of the diversity in the full HALOGAS sample. For each galaxy, we produced a continuum map created using all $10 \times 12 \mathrm{~h}$ of data, resulting in rms noise levels of about $30 \mu \mathrm{Jy}_{\text {beam }^{-1}}$. All targets are detected in continuum emission. We present maps of the total $\mathrm{H}_{\mathrm{I}}$ line intensity, comparisons with the optical image, and the Hi first-moment image or Renzogram.

For each galaxy we provide some discussion of the target and the results gleaned from our HALOGAS pilot survey data products. In the interest of brevity, we defer fuller detailed analyses of these and certain other individual HALOGAS targets (including NGC 4244; Zschaechner et al., in prep., UGC 4278; Serra et al., in prep., and NGC 949; Józsa et al., in prep.) to forthcoming papers. Ultimately, the main goal of the HALOGAS project is to examine the properties of extraplanar $\mathrm{H}_{\mathrm{I}}$ across the full sample; those results will be presented following the completion of all survey observations.

\subsection{UGC 2082}

UGC 2082 is an edge-on, low surface brightness galaxy which appears to be isolated (hence the lack of environmental information in the UGC 2082 entry in Table 2). It contains molecular gas (Matthews \& Gao 2001; Matthews et al. 2005), but has a low star formation rate. The SFR estimate listed in Table 1 $\left(0.023 M_{\odot} \mathrm{yr}^{-1}\right)$ is based on FIR luminosity, and by this estimator UGC 2082 has one of the lowest SFRs in our sample. We note, however, a confident detection of extended radio continuum emission from the inner disk.

With the HALOGAS $\mathrm{H}_{\mathrm{I}}$ observations, we detect a total $\mathrm{H}_{\mathrm{I}}$ mass of $M_{\mathrm{HI}}=2.54 \times 10^{9} M_{\odot}$. The vertical distribution of $\mathrm{H}_{\mathrm{I}}$ emission is somewhat extended, reaching up to a maximum vertical extent of about $5 \mathrm{kpc}$, fully one third of the radial extent of the H. The total H i contours along the major axis clearly show a slight warp in the sky plane, beginning somewhat beyond the optical radius. A warp component along the line of sight may be responsible for some of the apparent thickening of the disk.

Indeed, the appearance of the position-velocity (PV) diagram shown in Fig. 2 is suggestive of a line-of-sight warp. In this diagram, the major axis is located at zero angular offset. The characteristic triangular appearance, caused by emission at large vertical offsets appearing at velocities close to systemic, can be caused by a halo with lagging rotational velocities or by a line-of-sight warp (e.g. Oosterloo et al. 2007). In the case of UGC 2082, the visual appearance (in particular the narrowness of the velocity profiles at the largest angular offsets) is more similar to galaxies determined to show evidence for a line-of-sight warp (e.g., Rand \& Benjamin 2008; Gentile et al. 2003) rather than those determined to host a lagging halo. The Renzogram shown in Fig. 1 also shows characteristic signs of a line-of-sight warp: the contours of channels at intermediate velocities extend to larger radii with increasing distance from the midplane. That this effect is more pronounced on the approaching side, together with the uneven thickness of the $\mathrm{H}_{\mathrm{I}}$ distribution along the major axis, indicate that the line-of-sight warp is asymmetric. Geometrical modeling work is in progress to draw conclusions about how much of this vertical extension can be due to a halo, rather than warping (or flaring). Based on modeling as shown 

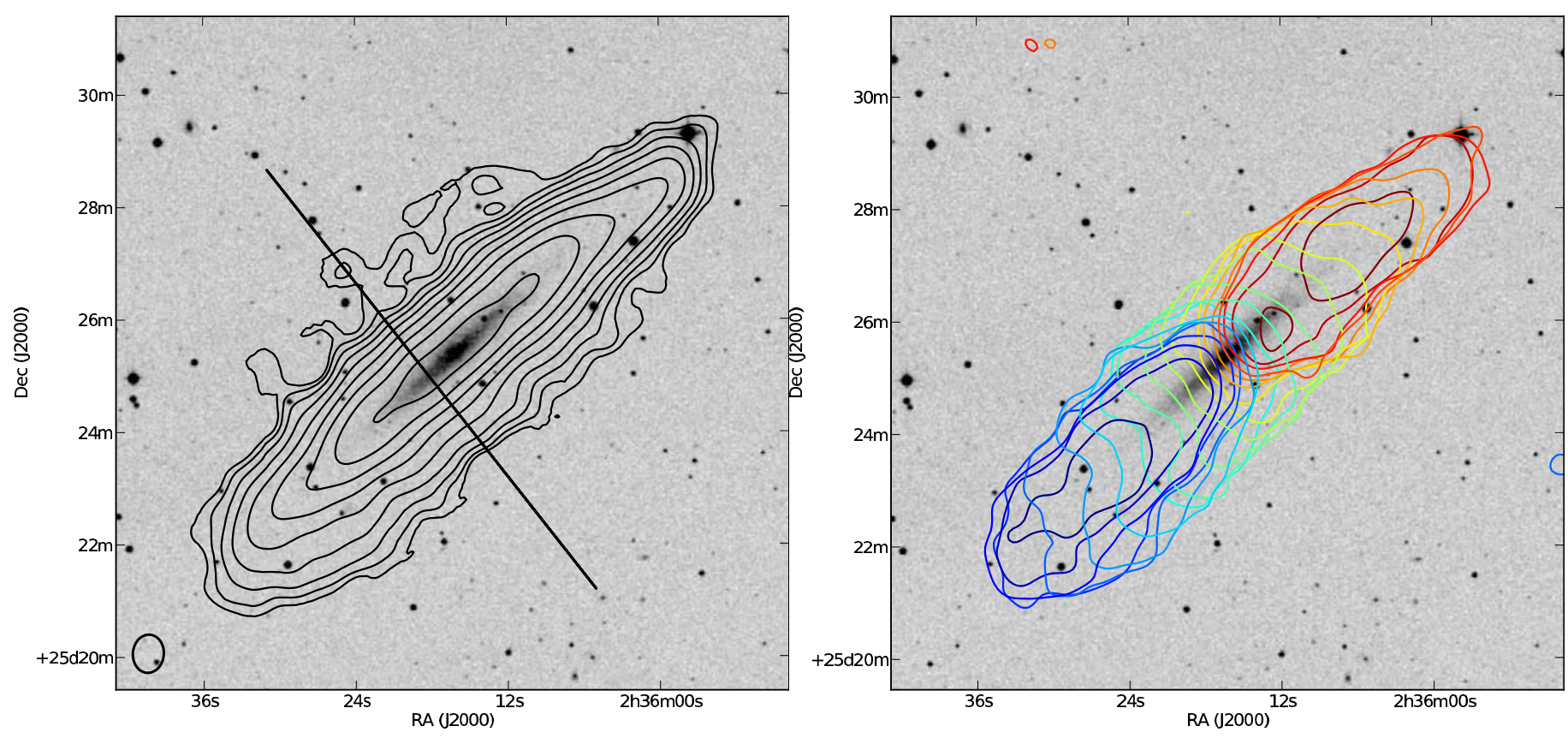

Fig. 1. Overview of the HALOGAS observations of UGC 2082. The left panel shows the H I total intensity overlaid on the DSS R-band image. The H I contours originate from the $30^{\prime \prime}$-tapered image, begin at $N_{\mathrm{HI}}=1.0 \times 10^{19} \mathrm{~cm}^{-2}$ and increase by powers of two. The straight line shows the orientation of the PV slice shown in Fig. 2. The right panel shows an overlay of several channels in the lowest resolution data cube, all at a level of $0.9 \mathrm{mJy}_{\text {beam }}^{-1}(\approx 3.75 \sigma)$. The contours are separated by $12.4 \mathrm{~km} \mathrm{~s}^{-1}$, begin at $593 \mathrm{~km} \mathrm{~s}^{-1}$ (dark blue) and range upward to $815 \mathrm{~km} \mathrm{~s}^{-1}$ (dark red). Both panels show the same area of the sky. The beam size of the $\mathrm{H}$ i data is shown in the lower left corners of the left panel.

by Swaters et al. (1997, their Fig. 4) and Oosterloo et al. (2007, their Fig. 12), the different situations can be distinguished by careful inspection of the individual channel maps, as well as PV diagrams such as the one shown in Fig. 2. A detailed description of the modeling work is beyond the scope of this pilot survey description paper, and it will instead be presented in a forthcoming publication.

It is unlikely that large-scale morphological features such as warping or flaring can explain all of the high-latitude $\mathrm{H}$ I emission, however. The "fingers" (filamentary features) of H I, extending upward perpendicular to the major axis at the largest $z$ on the northeast side, appear to be distinct enhancements of cold gas. One such feature is indicated with an arrow in Fig. 2. At a projected distance of $\approx 9 \mathrm{kpc}$ above the midplane, this cloud would have an $\mathrm{H}_{\mathrm{I}}$ mass of a few $10^{6} M_{\odot}$ and a broad velocity width of $60 \mathrm{~km} \mathrm{~s}^{-1}$. The presence of features like this one suggests that at least some of the thick disk of UGC 2082 is made up of a filamentary $\mathrm{H}$ i halo.

\subsection{NGC 672}

NGC 672 is a moderately inclined spiral which has a neighboring irregular galaxy, IC 1727. In Fig. 3, NGC 672 is the brighter galaxy centered in the frames, and IC 1727 is located to its southwest. At the adopted distance listed in Table $1(7.6 \mathrm{Mpc})$, the linear projected separation between the two galaxies is $16.3 \mathrm{kpc}$. In the optical image, there is no clear sign of a strong disturbance in NGC 672. Previous interferometric observations (Combes et al. 1980) have revealed a gravitational interaction with a substantial amount of gas in the interface region. In our observations, we detect a total mass of $3.78 \times 10^{9} M_{\odot}$, of which approximately $60 \%$ is associated with the primary, NGC 672. Both galaxies are clearly detected in continuum emission.

The Hi distribution shown in Fig. 3 reveals clearly the connection between the two galaxies. Each of the two galaxies

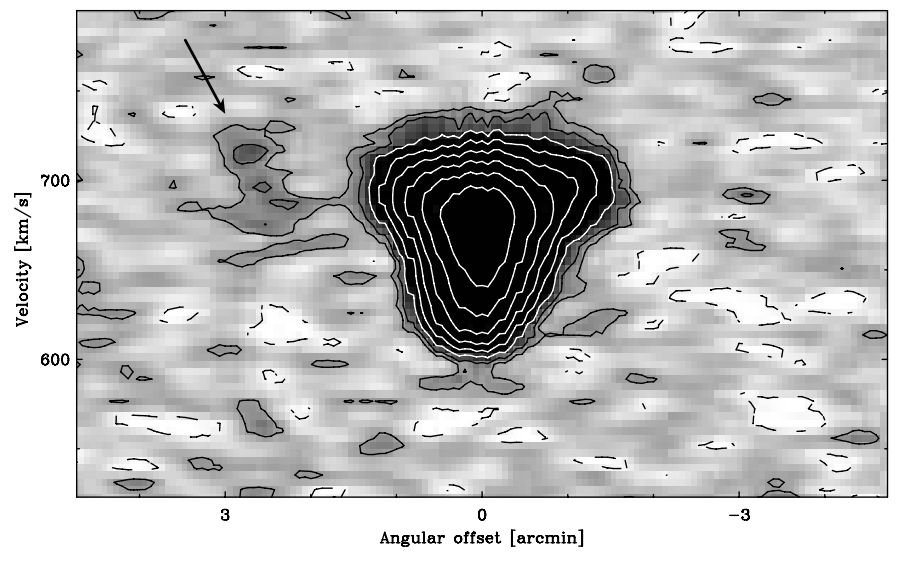

Fig. 2. Position-velocity (PV) diagram for UGC 2082, plotted using the $30^{\prime \prime}$-resolution cube. Contour levels begin at $300 \mu \mathrm{Jy} \mathrm{beam}^{-1}(\approx 1.5 \sigma$; see Table 3$)$ and increase by powers of two. The dashed contours are at $-300 \mu \mathrm{Jy} \mathrm{beam}^{-1}$. The line in Fig. 1 indicates the orientation of the PV slice shown here. The northeast end of the slice is to the left. At the distance listed in Table 1, $1^{\prime}$ corresponds to a linear scale of $3.11 \mathrm{kpc}$. The arrow indicates an extraplanar feature described in the text.

appears to be relatively undisturbed within their optical extent, but the outskirts and interface region are more complicated. The pair seems to be in an early stage of interaction. A clear enhancement of $\mathrm{H}_{\mathrm{I}}$ emission is located between the two galaxies, centered at about $\alpha_{\mathrm{J} 2000.0}=01^{\mathrm{h}} 47^{\mathrm{m}} 41^{\mathrm{s}}, \delta_{\mathrm{J} 2000.0}=27^{\mathrm{d}} 22^{\mathrm{m}} 10^{\mathrm{s}}$. To the northwest of the interaction region, a clear arc-shaped feature is seen extending away from the system. A faint diffuse extension is seen on the southwest side of IC 1727. A long, coherent armshaped feature starts just north of the center of NGC 672 and stretches away to the northeast. This could be a tidal or spiral arm, or may be a large $\mathrm{H}_{\mathrm{I}}$ loop seen in projection. 

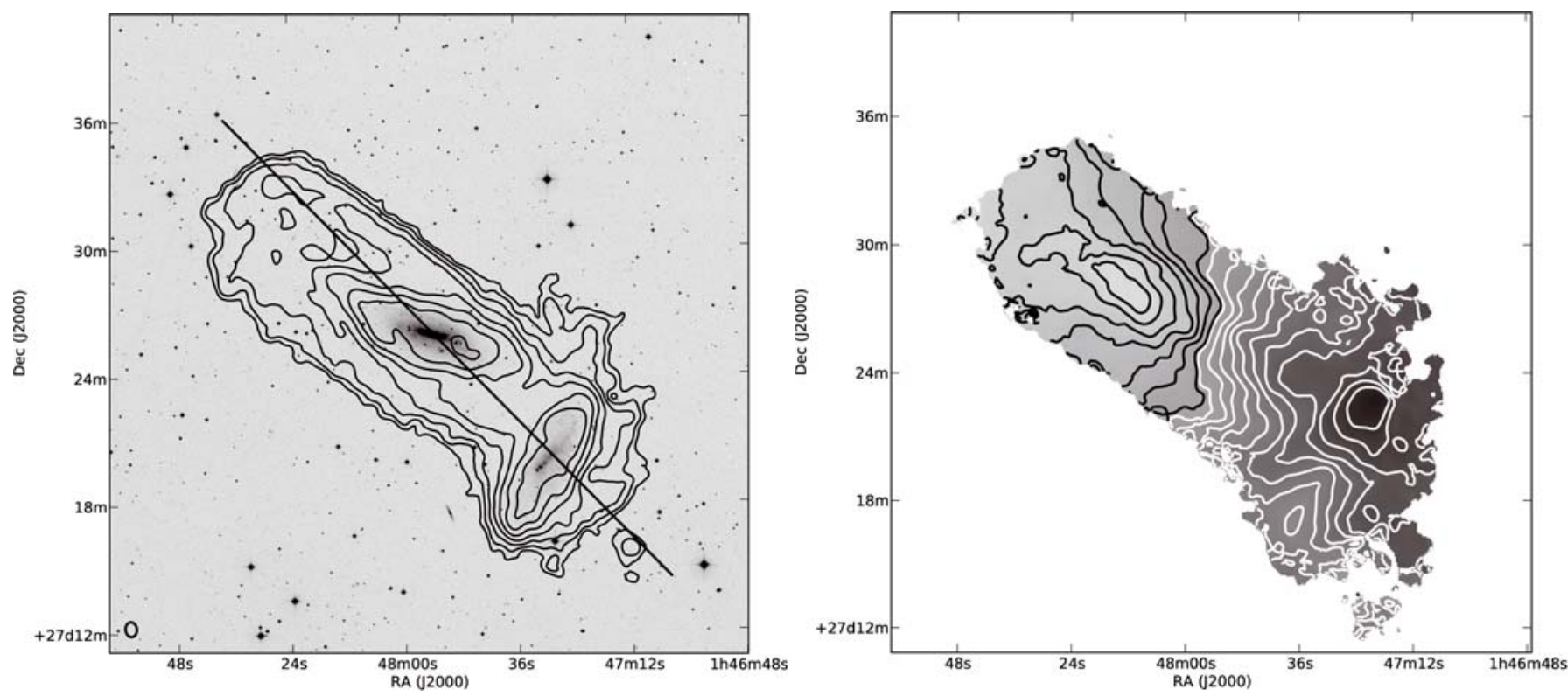

Fig. 3. Overview of the HALOGAS observations of NGC 672. The left panel is as in Fig. 1 . The contours begin at $N_{\mathrm{HI}}=2.5 \times 10^{19} \mathrm{~cm}^{-2}$ and increase by powers of two. The right panel shows the first-moment image derived from the lowest-resolution $\mathrm{H}$ i cube. The grayscale ranges from $245 \mathrm{~km} \mathrm{~s}^{-1}$ (black) to $570 \mathrm{~km} \mathrm{~s}^{-1}$ (white). Contours are white on the approaching side and black on the receding side, and run from $255 \mathrm{~km} \mathrm{~s} \mathrm{~s}^{-1}$ to $600 \mathrm{~km} \mathrm{~s}^{-1}$ in steps of $15 \mathrm{~km} \mathrm{~s}^{-1}$. The white contour closest to the center of the primary galaxy, corresponding to $425 \mathrm{~km} \mathrm{~s}^{-1}$, represents the systemic velocity of NGC 672.

The first-moment image indicates that the rotation of NGC 672 is fairly well ordered within the optical body on the northeast side (farther from the interaction region), and significantly more disturbed toward the companion galaxy. The main body of IC 1727 appears to show well ordered rotation. The gas in the system that is located outside of the optical disks also has kinematics which overall blend smoothly into the firstmoment images of the individual galaxies. It should be noted, however, that the velocity profiles are complicated in this region; this effect is clearly visible in the PV diagram shown in Fig. 4 (the emission near angular offsets of $3^{\prime}-4^{\prime}$ ). There, the profiles are significantly broadened, often have multiple peaks, and even show evidence for discrete emission at distinctly different velocities (e.g. the feature at angular offset $\sim 3^{\prime}$, and velocity $\left.420-460 \mathrm{~km} \mathrm{~s}^{-1}\right)$. For that reason, the mean velocities in the interface region shown in Fig. 3 should be regarded with suspicion. The PV diagram also shows that the systemic velocity of IC 1727 is consistent with a continuation of the kinematics of NGC 672. As noted by Sancisi et al. (2008), in many minor mergers the kinematics of the infalling companion galaxy follows that of the outer disk of the major galaxy.

In order to gain a better understanding of this system, we intend to use Identikit (Barnes \& Hibbard 2009), or similar software, to match the observations against computer models of galaxy interactions. This should give an indication of the threedimensional geometry of the system, as well as the interaction history. There is clearly a large quantity of unsettled gas in the system, which is potentially material that will be accreted at a later stage. The ultimate fate of this gas is of obvious interest.

\subsection{NGC 925}

NGC 925 is a moderately inclined spiral. We detect bright diffuse continuum emission, with enhancements corresponding to the locations of the most prominent features in the optical spiral arms. NGC 925 has been observed previously in the $\mathrm{HI}_{\mathrm{I}}$

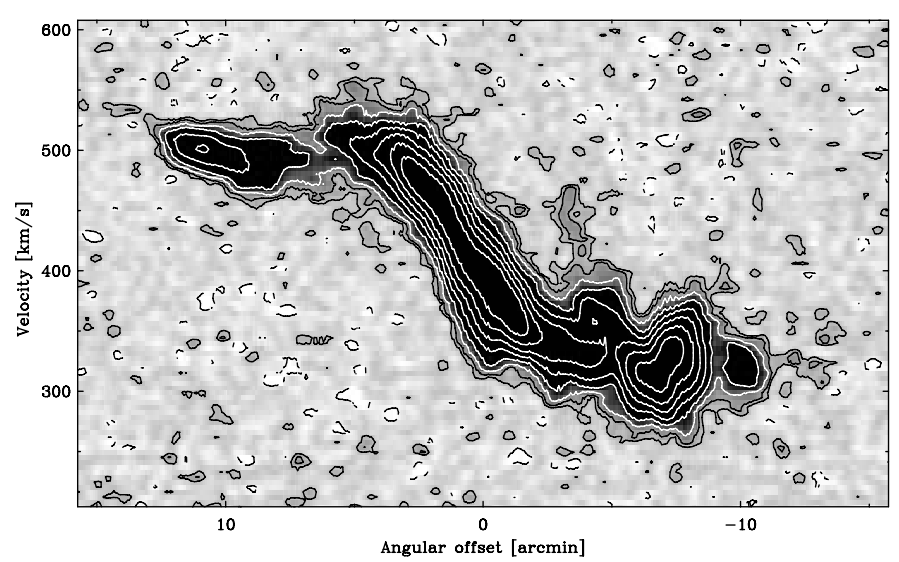

Fig. 4. As in Fig. 2, but for NGC 672. The line in Fig. 3 indicates the orientation of the PV slice shown here. The northeast end of the slice is to the left. At the distance listed in Table 1, 1' corresponds to a linear scale of $2.18 \mathrm{kpc}$.

line, notably by Pisano et al. (1998) and as part of THINGS (The H I Nearby Galaxy Survey; Walter et al. 2008). Neither of those studies detected $\mathrm{H}_{\mathrm{I}}$ at such large galactocentric radii as revealed in Fig. 5. Note that the image shown by Sancisi et al. (2008), based on earlier WSRT data, clearly illustrates the radially extended and disturbed $\mathrm{H}_{\mathrm{I}}$ distribution. With the HALOGAS observations, we recover the same structure, but with higher sensitivity. The inner disk within the optical body has an unsmooth appearance, with clumps and holes. Many of the $\mathrm{H}_{\mathrm{I}}$ overdensities correspond to the locations of bright knots of optical light in the spiral arms. The appearance of the outer disk of NGC 925 is that of a complicated stream of gas stripped during a minor merger. The twisting of the isovelocity contours in that region suggest outer disk warping or spiral structure. An enhancement in the H I column density is seen at the southernmost edge of the outer disk, at coordinates $\alpha_{\mathrm{J} 2000.0}=02^{\mathrm{h}} 26^{\mathrm{m}} 44^{\mathrm{s}}$, 

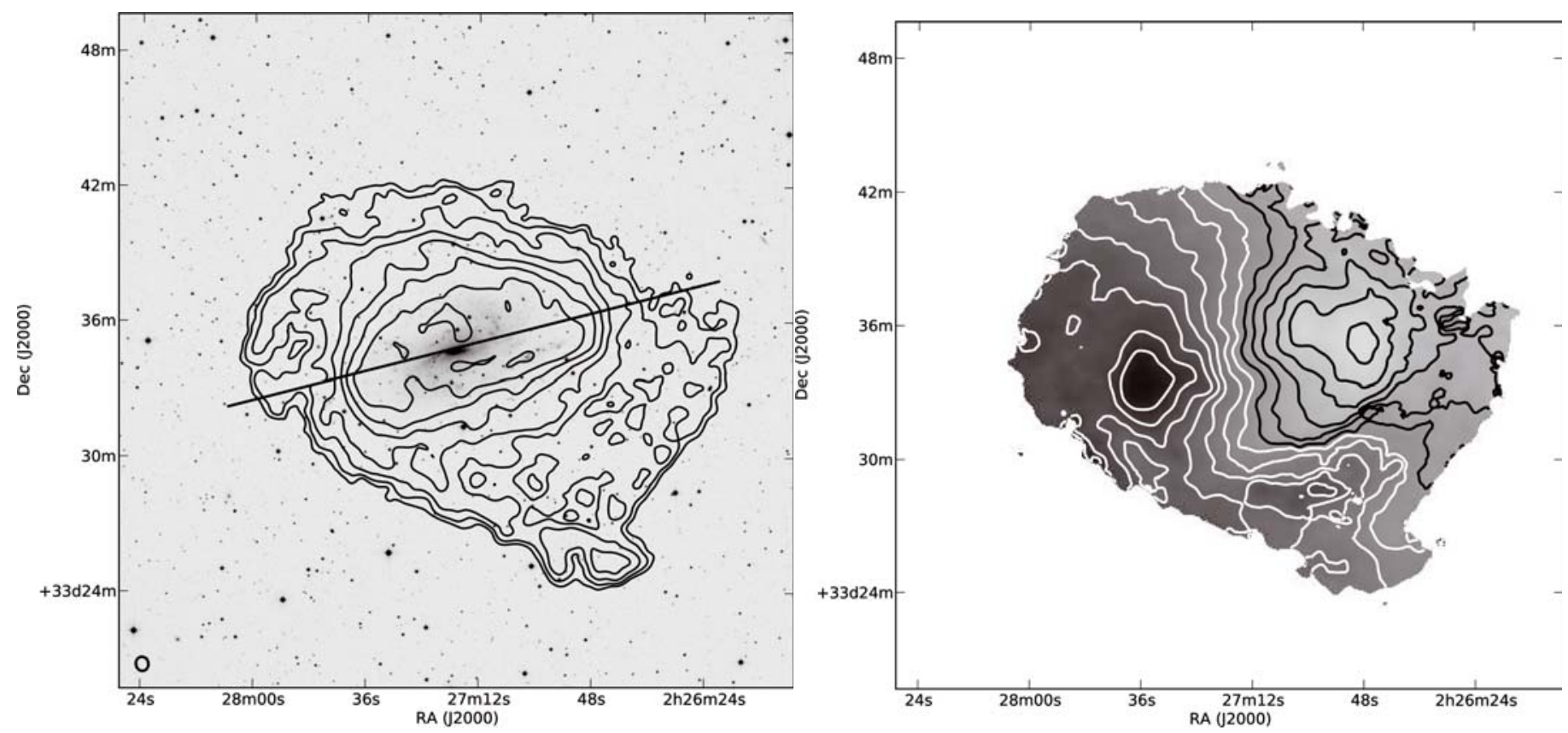

Fig. 5. Overview of the HALOGAS observations of NGC 925. The panels are as in Fig. 3 . The contours in the left panel begin at $N_{\mathrm{HI}}=2.0 \times$ $10^{19} \mathrm{~cm}^{-2}$ and increase by powers of two. The grayscale in the right panel ranges from $440 \mathrm{~km} \mathrm{~s}^{-1}$ (black) to $675 \mathrm{~km} \mathrm{~s}^{-1}$ (white). Contours are white on the approaching side and black on the receding side, and run from $450 \mathrm{~km} \mathrm{~s}^{-1}$ to $660 \mathrm{~km} \mathrm{~s}^{-1}$ in steps of $15 \mathrm{~km} \mathrm{~s}^{-1}$. The white contour closest to the center of the galaxy, corresponding to $555 \mathrm{~km} \mathrm{~s}^{-1}$, represents the systemic velocity of NGC 925 .

$\delta_{\mathrm{J} 2000.0}=33^{\mathrm{d}} 25^{\mathrm{m}} 20^{\mathrm{s}}$. Inspection of the optical DSS plates reveals that a faint fuzzy optical counterpart is very close to the location of this $\mathrm{H}_{\mathrm{I}}$ enhancement, centered at about $\alpha_{\mathrm{J} 2000.0}=$ $02^{\mathrm{h}} 26^{\mathrm{m}} 53^{\mathrm{s}}, \delta_{\mathrm{J} 2000.0}=33^{\mathrm{d}} 25^{\mathrm{m}} 40^{\mathrm{s}}$. Thus, the H $\mathrm{H}$ streams may be the result of the disruption of a gas-rich dwarf companion, whose stellar counterpart is still barely visible in the survey plates. NGC 925 is a prototypical minor merger, illustrating how gas is fed into large galaxies by the accretion of material from satellites.

With the HALOGAS observations we detect $5.89 \times 10^{9} M_{\odot}$ of $\mathrm{H}$ I. A large amount of anomalous $\mathrm{H}$ i emission is clearly visible in this target. While the streams in the outer disk generally follow the overall rotation of the inner disk (see the first-moment image in Fig. 5), there is also gas in the inner parts of the galaxy which either appear as "beard" emission (Fraternali et al. 2002), or sometimes even at distinct velocities relative to the bulk of the galaxy rotation. These features cannot be seen directly in the moment maps shown in Fig. 5. The beard gas is however readily visible in the PV diagram shown in Fig. 6. There, faint emission is seen to extend preferentially toward the systemic velocity (to lower velocity at positive angular offset, and higher velocity at negative angular offset). This may well be the sign of a slowly rotating halo seen in projection against the disk. Interpreted in this way, we estimate the total $\mathrm{H}_{\mathrm{I}}$ mass of the lagging component to be a few times $10^{8} M_{\odot}$, at a velocity difference of about $20-50 \mathrm{~km} \mathrm{~s}^{-1}$ with respect to the disk. We note that the SFR of NGC 925 is similar to that of NGC 2403, which is the prototypical galaxy demonstrating beard emission, and the latter is somewhat more massive. Detailed modeling of NGC 925 will allow us to separate the regularly rotating disk emission from the anomalous gas, determine its nature, and make a more detailed comparison with NGC 2403.

A small companion (outside of the frame in Fig. 5) is found to the north of NGC 925 at about $\alpha_{\mathrm{J} 2000.0}=02^{\mathrm{h}} 27^{\mathrm{m}} 20^{\mathrm{s}}, \delta_{\mathrm{J} 2000.0}=$ $33^{\mathrm{d}} 57^{\mathrm{m}} 30^{\mathrm{s}}$. Its $\mathrm{H}_{\mathrm{I}}$ mass is $1.27 \times 10^{7} M_{\odot}$. This object, too, appears to have a faint optical counterpart in the DSS plates. It is located at a projected distance of $60 \mathrm{kpc}$ from the center of NGC 925 and does not show any obvious signs of interaction.

\subsection{NGC 4565}

NGC 4565 is a large, edge-on spiral galaxy which is similar to the Milky Way. The new HALOGAS observations are combined with archival data presented by Dahlem et al. (2005). The other two galaxies in the frame are NGC 4562, a small companion classified in the RC3 as type $\mathrm{SB}(\mathrm{s}) \mathrm{dm}$ and visible to the southwest of NGC 4565, and IC 3571, the small galaxy located very close to NGC 4565 on its north side. In the PV diagram shown in Fig. 8, the vertically extended emission in NGC 4565 is seen to have a smooth velocity gradient directly toward the systemic velocity of IC 3571 .

The distribution of $\mathrm{Hr}$ emission is shown in Fig. 7. The total $\mathrm{H}_{\mathrm{I}}$ mass detected in NGC 4565 itself is $7.43 \times 10^{9} M_{\odot}$. The galaxy is obviously warped: this can be seen in the total H I map (particularly obvious on the north end of the disk) and also in the first-moment image contours. NGC 4562 has a regularly rotating

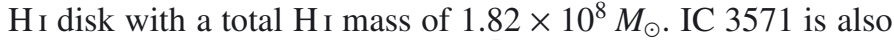
detected in $\mathrm{H}_{\mathrm{I}}\left(M_{\mathrm{HI}}=4.18 \times 10^{7} M_{\odot}\right)$, and the $\mathrm{H}_{\mathrm{I}}$ data show clear signs of a minor interaction between it and NGC 4565.

NGC 4565 has a moderately high star formation rate when compared to the rest of the HALOGAS sample. The most similar HALOGAS sample galaxy (with existing observations to date) is NGC 891. There are, however, several key differences between these two galaxies. First, the SFR of NGC 4565 is only about one quarter of that of NGC 891. Both galaxies have high rotation speeds (but that of NGC 4565 is $15 \%$ higher - it has the highest rotation speed of all the HALOGAS sample galaxies). The distances to NGC 4565 and NGC 891, and their optical diameters, indicate that NGC 4565 is larger in linear dimensions.

It is apparent from Fig. 7 that the $\mathrm{H}_{\mathrm{I}}$ distribution in this galaxy is far less vertically extended than that of NGC 891 (compare with Fig. 1 of Oosterloo et al. 2007), even though our new 


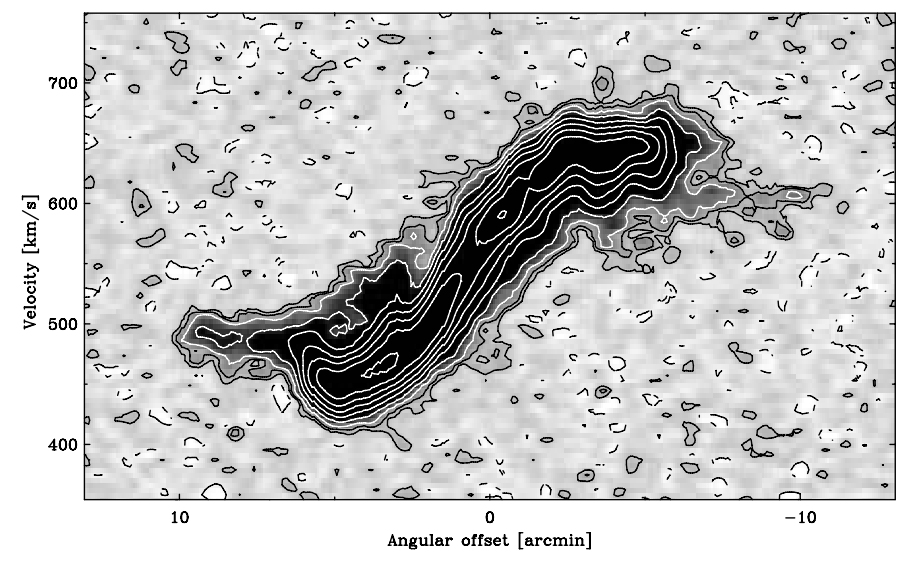

Fig. 6. As in Fig. 2, but for NGC 925. The line in Fig. 5 indicates the orientation of the PV slice shown here. The east end of the slice is to the left. The lowest contour level (solid and dashed) is $\pm 250 \mu \mathrm{Jy}_{\text {beam }}{ }^{-1}$. At the distance listed in Table $1,1^{\prime}$ corresponds to a linear scale of $2.73 \mathrm{kpc}$.

observations have comparable sensitivity to the NGC 891 work. Although the disk of NGC 4565 is clearly somewhat warped (and importantly, also partially along the line of sight; Rupen 1991), we can still derive a reasonable upper limit to the vertical extent, of approximately $5 \mathrm{kpc}$ (using PA $=315^{\circ}$; Dahlem et al. 2005). Meanwhile, the Hi halo of NGC 891 extends up to more than $10 \mathrm{kpc}-\mathrm{a}$ distinct difference. Previous work has also determined that there is a lack of ionized gas in the halo of NGC 4565 (see Rand et al. 1992). A final difference between the two galaxies is that NGC 891 has a extremely bright and vertically extended radio continuum halo (e.g., Dahlem et al. 1994). We find that NGC 4565 also has a vertically extended, and to our knowledge previously undetected at these frequencies, radio continuum halo (see Fig. 9). However, it does not seem to be as extended (the continuum halo in NGC 4565 reaches up to 2-3 kpc, compared to $10 \mathrm{kpc}$ in NGC 891), nor is it as bright, as the halo in NGC 891. Studies of the continuum emission (and its polarization) in NGC 4565 are described by Hummel et al. (1984) and Sukumar \& Allen (1991). Further discussion regarding the differences between NGC 4565 and NGC 891 is provided in Sect. 5.

\section{Discussion}

The HALOGAS pilot survey, while still limited in the number of targets, nevertheless covers a substantial range of galactic properties. From the perspective of environmental properties, we have an apparently isolated galaxy (UGC 2082), two targets that have small, gas-rich companions (NGC 925 and NGC 4565), and one galaxy in an interacting pair (NGC 672). We also sample about two orders of magnitude in star formation rate, and nearly a factor of three in rotation speed, with this pilot survey. While it is clear that none of the pilot survey galaxies have an H I halo of the scale seen in NGC 891, we find that all of the pilot survey targets show varying indications of cold gas in their halos.

The lack of a prominent halo in UGC 2082 may not be a surprising result: the star formation rate is low, and the galaxy is isolated. Apart from the possibility of significant primordial accretion, a ready source for creating a gaseous halo does not seem to be present in this target. Nonetheless, we find several distinct extraplanar features (these are noted in Sect. 4.1 and are visible in Figs. 1 and 2). These clouds may be a sign of cold gas accretion.

Two of the pilot galaxies that are located in denser environments have a much less regular H i structure. Clearly, the case of NGC 672 is a complicated one - the strong interaction with its companion IC 1727 has spread H I emission throughout the system. This is a dramatic case of interaction-induced accretion. Another interacting system but at a later stage, NGC 925 seems to have interacted with a gas-rich companion sometime in its recent past, resulting in stripped material surrounding the main disk. Whether the culprit is the faint blob at the southern edge of the galaxy, the object found to the north (as described in Sect. 4.3), or some other entity, is not yet clear. NGC 925 also seems to possess $\mathrm{H}$ i at lagging velocities with respect to the main disk. The anomalous component in NGC 925 has a similar mass and kinematic lag when compared to the same feature in NGC 2403.

Another target to compare with existing observations is the large edge-on NGC 4565, which does not show evidence of a prominent halo component. Can this lack be explained? In comparison with NGC 891, the differences in the global parameters of the two galaxies all tend to be in the sense in which one would indeed expect a larger halo in NGC 891, if gaseous halos are induced primarily by star formation-driven galactic fountain flows. Under that assumption, the most important properties of a galaxy for determining whether a halo is formed should be (1) the SF energy injection rate (per unit disk area; see e.g. Dahlem et al. 1995; Rossa \& Dettmar 2003); and (2) the gravitational potential (traced by $v_{\text {rot }}^{2}$ ). With NGC 891's larger SFR, smaller disk area (at the distances listed in Table 1), and lower rotational velocity, it would seem that it should indeed be more efficient in maintaining a gaseous halo. Per unit disk area, more star formation energy is available, and gravity is less effective at preventing material from moving away from the disk midplane. That NGC 925 and NGC 2403 have similar anomalous gas features, as well as similar SFR and mass, would also be consistent with such a picture of gaseous halo formation.

Do the other HALOGAS targets follow the same trend with the global galaxy parameters? Though it is intriguing, the number of galaxies which have been observed with enough sensitivity to distinguish a population of halo gas remains rather low, so that general statements are still uncertain. This initial result from our pilot survey underlines the need for a suitably large survey, in order to trace a range of variables in the population of nearby galaxies. The full HALOGAS survey will provide such a large sample.

\section{Multi-wavelength supplements}

For a full census of the contents of the survey galaxies, our $\mathrm{H}$ I observations must be supplemented with high-quality, sensitive observations tracing the distribution and kinematics of stars, dust, and other gas phases. With that in mind, the WSRT HALOGAS program is being supplemented in several ways.

Since minor mergers are thought to be one of the major channels for gas accretion, stellar- and gas accretion are profoundly linked and cannot be studied independently. We therefore obtained observing time with the Isaac Newton Telescope (INT; using the Wide Field Camera) in order to complement the deep HALOGAS H I observations with a photometric survey suited to detect the stellar remnants of former accretion events. The INT observations for a subset of the HALOGAS sample have been obtained, and the data will be presented in a 

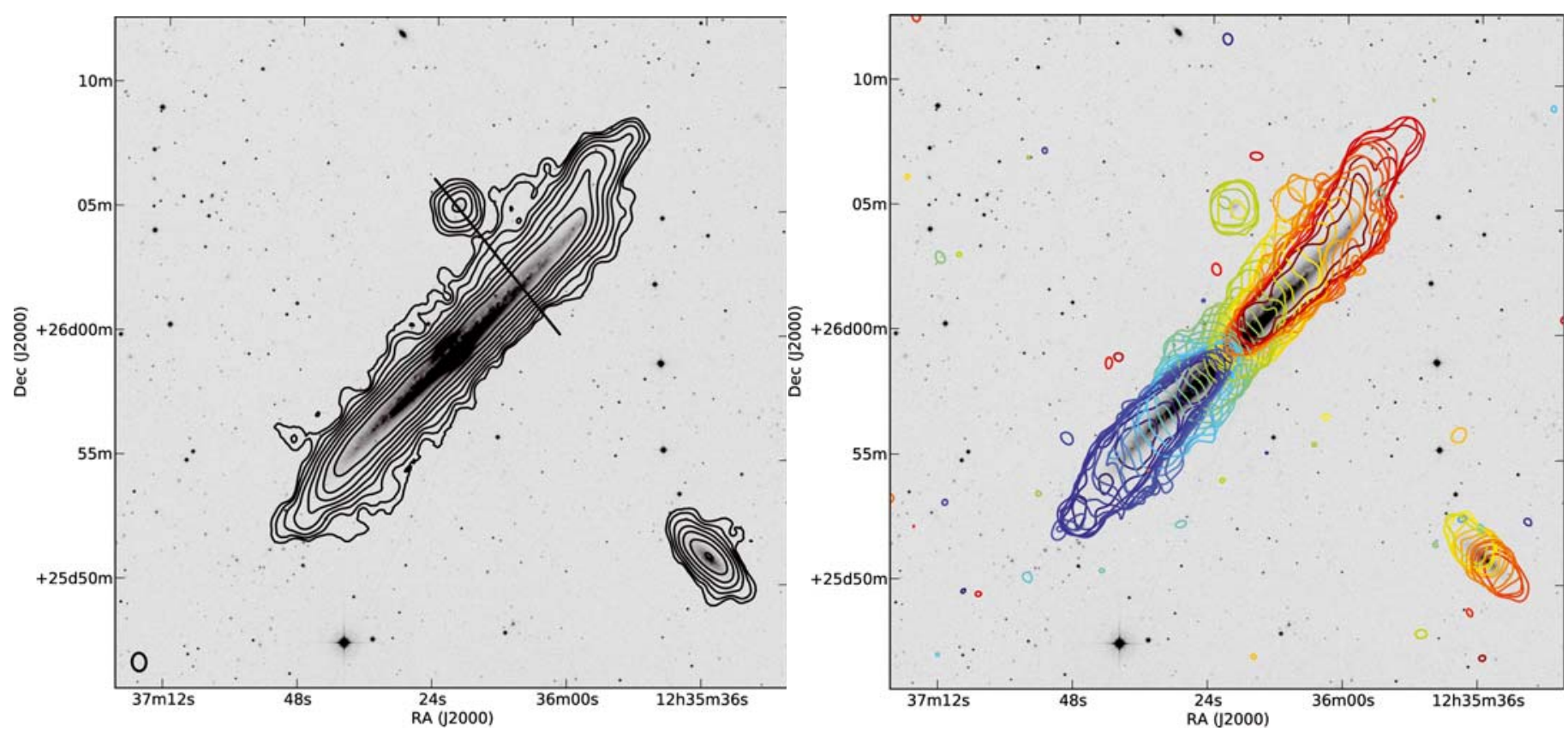

Fig. 7. Overview of the HALOGAS observations of NGC 4565. The panels are as in Fig. 1 . The contours in the left panel begin at $N_{\mathrm{HI}}=$

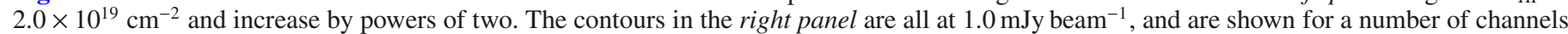
separated by $12.4 \mathrm{~km} \mathrm{~s}^{-1}$, starting at $945 \mathrm{~km} \mathrm{~s}^{-1}$ (dark blue) and ranging upwards to $1489 \mathrm{~km} \mathrm{~s}^{-1}$ (dark red).

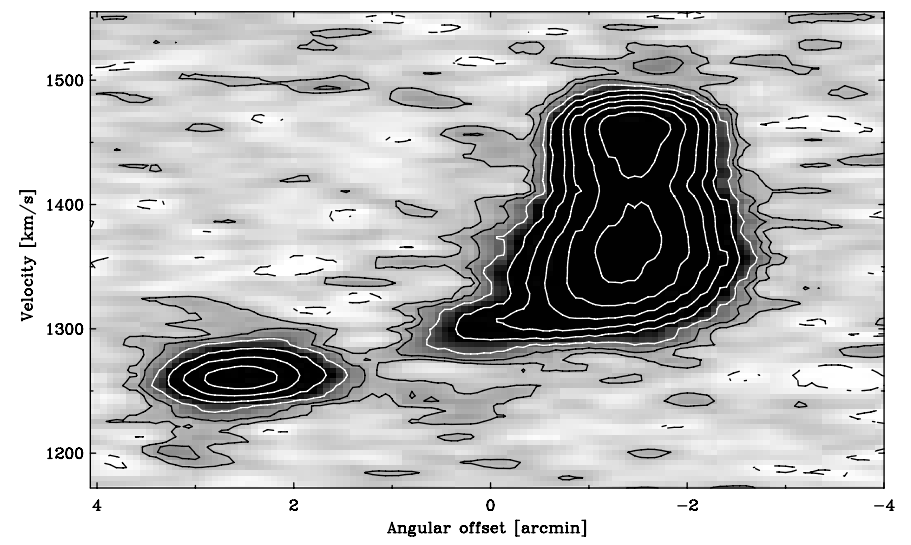

Fig. 8. As in Fig. 2, but for NGC 4565. The line in Fig. 7 indicates the orientation of the PV slice shown here. The northeast end of the slice is to the left. The lowest contour level (solid and dashed) is $\pm 350 \mu \mathrm{Jy}_{\text {beam }}{ }^{-1}$. At the distance listed in Table $1,1^{\prime}$ corresponds to a linear scale of $2.82 \mathrm{kpc}$.

forthcoming companion paper. This optical observing program is named HALOSTARS.

The $3.5 \mathrm{~m}$ ARC telescope at Apache Point Observatory is being used for kinematic observations of the ionized gas in a sample which overlaps the edge-on subsample of HALOGAS, using a novel multi-slit technique that allows for simultaneous $\mathrm{H} \alpha$ spectra of 11 parallel long slits, spaced $22^{\prime \prime}$ apart over a $4.5^{\prime} \times 4.5^{\prime}$ field of view. Together this set of long slits can be used to form a velocity field. So far, data have been obtained for NGC 891, NGC 3044 (not a HALOGAS target), NGC 4244, NGC 4565, and NGC 4631. The observations will continue through the next several observing seasons. These optical observations will allow us to test whether both the ionized and neutral phases share the same origin and kinematics (as has been previously investigated in NGC 891; Heald et al. 2006). Moreover, the ionized component is more tightly coupled to the radial extent of the star

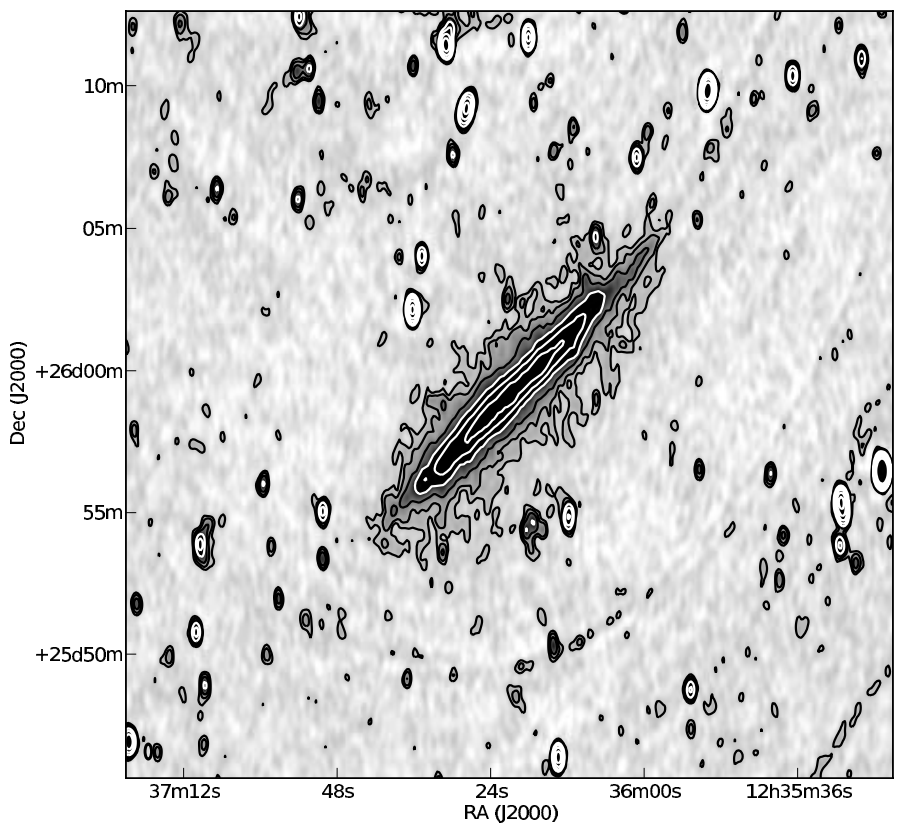

Fig. 9. Radio continuum image of NGC 4565. The contour levels begin at $54 \mu \mathrm{Jy}_{\text {beam }}^{-1}(\approx 3 \sigma)$ and increase by powers of two.

forming disk than is the neutral, likely resulting in little to no contamination from warped outer disk gas complicating the analysis of extraplanar emission. This provides an important check on the $\mathrm{H}$ I modeling results.

We have received $102 \mathrm{ks}$ on the Galaxy Evolution Explorer (GALEX; Martin et al. 2005) satellite to observe 8 galaxies, of which 6 are drawn from the HALOGAS sample and 2 from a complementary Very Large Array (VLA) H i program. In addition, 3 HALOGAS targets are already being observed as part of the GALEX Deep Galaxy Survey (DGS) survey, while NGC 2403 and NGC 891 also have existing deep archival 
GALEX exposures from other programs. We were granted observations in both GALEX bands, but unfortunately the FUV channel onboard GALEX is now unavailable, so that we will only obtain NUV images. The purposes of the GALEX observations are $(i)$ to search for scattered light from dust co-located with the gaseous halo in edge-on systems, which would help elucidate the origin of this material; and (ii) to perform a highly sensitive search for star formation in the outer disks and halo regions in all of the galaxies, allowing us to study the correlation between gas accretion and outer disk star formation.

Finally, we also plan on obtaining wide-field UBRH $\alpha$ imaging in support of the GALEX and WSRT observations to search for outer disk and halo star formation, and for companions to determine to what extent accretion of gas may be associated with gas rich faint dwarfs.

\section{Data release and availability}

The calibrated survey data will be made publicly available in stages as the observations, data reduction, and analysis progress. The survey webpage is located at http://www.astron.nl/halogas and this is the primary location at which reduced data cubes, moment maps, and other data products can be found. Data releases will coincide with the publication of analysis papers. Pre-release data products may be obtained by contacting the HALOGAS team.

Acknowledgements. The Westerbork Synthesis Radio Telescope is operated by ASTRON (Netherlands Institute for Radio Astronomy) with support from the Netherlands Foundation for Scientific Research (NWO). This material is based in part upon work supported by the National Science Foundation under Grant No. AST 0908106 to RJR and AST 0908126 to RAMW. R.A.M.W. also acknowledges support from Research Corporation for this project. G.G. is a postdoctoral researcher of the FWO-Vlaanderen (Belgium). We acknowledge the usage of the HyperLeda database (http://leda.univ-lyon1.fr) in the course of constructing the survey sample. This research has made use of the NASA/IPAC Extragalactic Database (NED) which is operated by the Jet Propulsion Laboratory, California Institute of Technology, under contract with the National Aeronautics and Space Administration.

\section{References}

Barnabè, M., Ciotti, L., Fraternali, F., \& Sancisi, R. 2006, A\&A, 446, 61 Barnes, J. E., \& Hibbard, J. E. 2009, AJ, 137, 3071

Belokurov, V., Evans, N. W., Irwin, M. J., et al. 2007, ApJ, 658, 337

Benjamin, R. A. 2000, in Rev. Mex. Astron. Astrofis. Conf. Ser., ed. S. J. Arthur,

N. S. Brickhouse, \& J. Franco, 9, 256

Binney, J., Dehnen, W., \& Bertelli, G. 2000, MNRAS, 318, 658

Boomsma, R., Oosterloo, T. A., Fraternali, F., van der Hulst, J. M., \& Sancisi, R. 2008, A\&A, 490, 555

Bournaud, F., Combes, F., Jog, C. J., \& Puerari, I. 2005, A\&A, 438, 507

Bregman, J. N. 1980, ApJ, 236, 577

Chiappini, C., Matteucci, F., \& Gratton, R. 1997, ApJ, 477, 765

Combes, F., Foy, F. C., Weliachew, L., \& Gottesman, S. T. 1980, A\&A, 84, 85

Corbelli, E., \& Bandiera, R. 2002, ApJ, 567, 712

Dahlem, M., Dettmar, R., \& Hummel, E. 1994, A\&A, 290, 384

Dahlem, M., Lisenfeld, U., \& Golla, G. 1995, ApJ, 444, 119

Dahlem, M., Ehle, M., Ryder, S. D., Vlajić, M., \& Haynes, R. F. 2005, A\&A, 432, 475

Dale, D. A., \& Helou, G. 2002, ApJ, 576, 159

Dale, D. A., Cohen, S. A., Johnson, L. C., et al. 2009, ApJ, 703, 517

Ferguson, A. M. N. 2007, in From Stars to Galaxies: Building the Pieces to Build

Up the Universe, ed. A. Vallenari, R. Tantalo, L. Portinari, \& A. Moretti, ASP Conf. Ser., 374, 239
Fraternali, F., \& Binney, J. J. 2008, MNRAS, 386, 935

Fraternali, F., van Moorsel, G., Sancisi, R., \& Oosterloo, T. 2002, AJ, 123, 3124 Fraternali, F., Oosterloo, T. A., Sancisi, R., \& Swaters, R. 2005, in Extra-Planar Gas, ed. R. Braun, ASP Conf. Ser., 331, 239

Gentile, G., Fraternali, F., Klein, U., \& Salucci, P. 2003, A\&A, 405, 969

Giuricin, G., Marinoni, C., Ceriani, L., \& Pisani, A. 2000, ApJ, 543, 178

Hameed, S., \& Devereux, N. 2005, AJ, 129, 2597

Heald, G., \& Oosterloo, T. A. 2008, ed. J. G. Funes, \& E. M. Corsini, in ASP Conf. Ser., 396, 267

Heald, G. H., Rand, R. J., Benjamin, R. A., \& Bershady, M. A. 2006, ApJ, 647, 1018

Heald, G. H., Rand, R. J., Benjamin, R. A., \& Bershady, M. A. 2007, ApJ, 663, 933

Hummel, E., Sancisi, R., \& Ekers, R. D. 1984, A\&A, 133, 1

Józsa, G. I. G., Kenn, F., Klein, U., \& Oosterloo, T. A. 2007, A\&A, 468, 731

Kamphuis, P., Peletier, R. F., Dettmar, R., et al. 2007, A\&A, 468, 951

Kaufmann, T., Mayer, L., Wadsley, J., Stadel, J., \& Moore, B. 2006, MNRAS, 370,1612

Kennicutt, R. C., Hao, C., Calzetti, D., et al. 2009, ApJ, 703, 1672

Kennicutt, Jr., R. C. 1998, ApJ, 498, 541

Kennicutt, Jr., R. C., Armus, L., Bendo, G., et al. 2003, PASP, 115, 928

Kennicutt, Jr., R. C., Lee, J. C., Funes, G. S. J. J., Sakai, S., \& Akiyama, S. 2008, ApJS, 178, 247

Kereš, D., Katz, N., Weinberg, D. H., \& Davé, R. 2005, MNRAS, 363, 2

Lacey, C. G., \& Fall, S. M. 1985, ApJ, 290, 154

Larson, R. B., Tinsley, B. M., \& Caldwell, C. N. 1980, ApJ, 237, 692

Lee, J. C., Kennicutt, R. C., Funes, José, G. S. J. J., Sakai, S., \& Akiyama, S. 2007, ApJ, 671, L113

Lisenfeld, U., Verdes-Montenegro, L., Sulentic, J., et al. 2007, A\&A, 462, 507

Marinacci, F., Binney, J., Fraternali, F., et al. 2010a, MNRAS, 347

Marinacci, F., Fraternali, F., Ciotti, L., \& Nipoti, C. 2010b, MNRAS, 401, 2451

Martin, D. C., Fanson, J., Schiminovich, D., et al. 2005, ApJ, 619, L1

Matthews, L. D., \& Gao, Y. 2001, ApJ, 549, L191

Matthews, L. D., \& Wood, K. 2003, ApJ, 593, 721

Matthews, L. D., Gao, Y., Uson, J. M., \& Combes, F. 2005, AJ, 129, 1849

Moshir, M. et al. 1990, in IRAS Faint Source Catalogue, version 2.0

Mouhcine, M., Ibata, R., \& Rejkuba, M. 2010, ApJ, 714, L12

Moustakas, J., \& Kennicutt, Jr., R. C. 2006, ApJS, 164, 81

Norman, C. A., \& Ikeuchi, S. 1989, ApJ, 345, 372

Oosterloo, T., Fraternali, F., \& Sancisi, R. 2007, AJ, 134, 1019

Pagel, B. E. J. 1997, Nucleosynthesis and Chemical Evolution of Galaxies, ed. B. E. J. Pagel

Paturel, G., Petit, C., Prugniel, P., et al. 2003, A\&A, 412, 45

Pisano, D. J., Wilcots, E. M., \& Elmegreen, B. G. 1998, AJ, 115, 975

Rand, R. J. 1996, ApJ, 462, 712

Rand, R. J., \& Benjamin, R. A. 2008, ApJ, 676, 991

Rand, R. J., Kulkarni, S. R., \& Hester, J. J. 1992, ApJ, 396, 97

Rice, W., Lonsdale, C. J., Soifer, B. T., et al. 1988, ApJS, 68, 91

Robitaille, T. P., Rossa, J., Bomans, D. J., \& van der Marel, R. P. 2007, A\&A, 464, 541

Rossa, J., \& Dettmar, R. 2003, A\&A, 406, 493

Rupen, M. P. 1991, AJ, 102, 48

Sancisi, R., Fraternali, F., Oosterloo, T., \& van der Hulst, T. 2008, A\&ARv, 15, 189

Sanders, D. B., Mazzarella, J. M., Kim, D., Surace, J. A., \& Soifer, B. T. 2003, AJ, 126, 1607

Sault, R. J., Teuben, P. J., \& Wright, M. C. H. 1995, in Astronomical Data Analysis Software and Systems IV, ed. R. A. Shaw, H. E. Payne, \& J. J. E. Hayes, ASP Conf. Ser., 77, 433

Schönrich, R., \& Binney, J. 2009, MNRAS, 396, 203

Struck, C., \& Smith, D. C. 2009, MNRAS, 398, 1069

Sukumar, S., \& Allen, R. J. 1991, ApJ, 382, 100

Swaters, R. A., Sancisi, R., \& van der Hulst, J. M. 1997, ApJ, 491, 140

Thilker, D. A., Braun, R., Walterbos, R. A. M., et al. 2004, ApJ, 601, L39

Tripp, T. M., Wakker, B. P., Jenkins, E. B., et al. 2003, AJ, 125, 3122

Tully, R. B. 1988, Nearby galaxies catalog, ed. R. B. Tully

Twarog, B. A. 1980, ApJ, 242, 242

Wakker, B. P., York, D. G., Howk, J. C., et al. 2007, ApJ, 670, L113

Wakker, B. P., York, D. G., Wilhelm, R., et al. 2008, ApJ, 672, 298

Walter, F., Brinks, E., de Blok, W. J. G., et al. 2008, AJ, 136, 2563

Wright, E. L. 2008, in EAS Publ. Ser. 33, ed. H. Zinnecker, N. Epchtein, \& H. Rauer, 57 